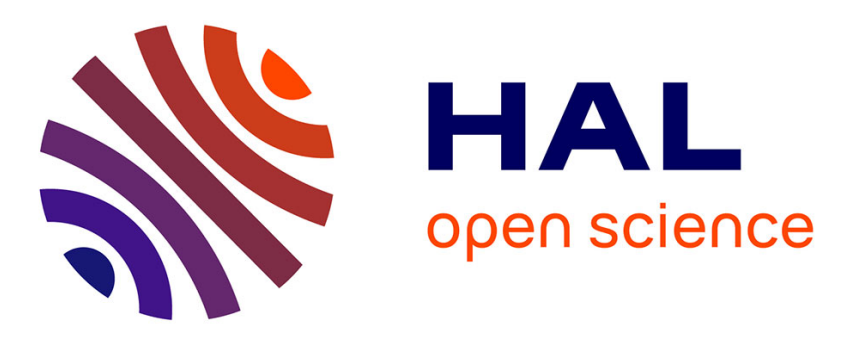

\title{
Excitation of Sidebands Due to Nonlinear Coupling Between a VLF Transmitter Signal and a Natural ELF Emission
}

V I Sotnikov, V Fiala, François Lefeuvre, Dominique Lagoutte, France M

Mogilevsky

\section{To cite this version:}

V I Sotnikov, V Fiala, François Lefeuvre, Dominique Lagoutte, France M Mogilevsky. Excitation of Sidebands Due to Nonlinear Coupling Between a VLF Transmitter Signal and a Natural ELF Emission. Journal of Geophysical Research Space Physics, 1991, 96 (A7), pp.363-374. 10.1029/91JA00695 . insu-02880037

\section{HAL Id: insu-02880037 https://hal-insu.archives-ouvertes.fr/insu-02880037}

Submitted on 24 Jun 2020

HAL is a multi-disciplinary open access archive for the deposit and dissemination of scientific research documents, whether they are published or not. The documents may come from teaching and research institutions in France or abroad, or from public or private research centers.
L'archive ouverte pluridisciplinaire HAL, est destinée au dépôt et à la diffusion de documents scientifiques de niveau recherche, publiés ou non, émanant des établissements d'enseignement et de recherche français ou étrangers, des laboratoires publics ou privés. 


\title{
Excitation of Sidebands Due to Nonlinear Coupling Between a VLF Transmitter Signal and a Natural ELF Emission
}

\author{
V. I. SOTNIKov, ${ }^{1}$ V. Fiala, ${ }^{2}$ F. LefeUVRe, AND D. LAGOUTTE \\ Centre National de la Recherche Scientifique, Laboratoire de Physique et Chimie de l'Environnement, Orleans,
} France

\section{MOGILEVSKY}

Space Research Institute, USSR Academy of Sciences, Moscow

\begin{abstract}
Symmetric sidebands are observed in the ionosphere by the AUREOL 3 satellite when it passes at a height of $1200 \mathrm{~km}$ above the VLF transmitter at the Komsomolsk-on-Amur Alpha station $\left(50^{\circ} 5 \mathrm{~N}\right.$, $135^{\circ} \mathrm{E}$, frequency 11.90 and $12.65 \mathrm{kHz}$ ). The sidebands are about $500 \mathrm{~Hz}$ off the carrier frequency of Alpha pulses. They are approximately $20 \mathrm{~dB}$ lower than the transmitter signal, and they appear only when ELF natural emission above the local proton gyrofrequency is observed. The data are presented and analyzed. The nonlinear coupling of the VLF transmitter signal to natural ELF emission is invoked to explain the symmetric sidebands. It is shown that the nonlinear current excited by the beats of VLF and ELF waves is strong enough to explain the sideband amplitude.
\end{abstract}

\section{INTRODUCTION}

Nearly monochromatic signals injected from ground-based VLF transmitters are known to experience bandwidth expansion as they traverse the ionosphere [Bell et al., 1983; Titova et al., 1984; Inan and Bell, 1985; Tanaka et al., 1987; Chmyrev et al., 1989] and the magnetosphere [Bell and Ngo, 1988 ] at low $L$ values ( $L \leq 4)$. Assuming the existence of magnetic-field-aligned plasma density irregularities, several authors have developed source models based upon linear [Titova et al., 1984; Bell and Ngo, 1988, 1990] and nonlinear [Groves et al., 1988] scattering. In the absence of ionospheric irregularities a mechanism based on a parametric instability was proposed [Riggin and Kelley, 1982; Lee and Kuo, 1984; Groves et al., 1988]. It excites a magnetic-field-aligned purely growing mode, together with one or two sidebands of lower hybrid waves.

Reports on sideband signals associated with VLF transmitter signals are rather scarce. Spectral peaks have been identified near the magnetic equatorial plane on the ISEE satellite at approximately plus and minus $55 \mathrm{~Hz}$ of the carrier frequency (13.1 and $13.6 \mathrm{kHz}$ ) of Omega pulses [Bell, 1985]. They are reduced in amplitude by about $5 \mathrm{~dB}$ with respect to the carrier frequency. Similar peaks seem to be present in the broadened spectra observed on the COSMOS 1809 satellite and generated in the ionosphere by the carrier frequency (19.1 $\mathbf{k H z}$ ) of UPD8 pulses [Chmyrev et al., 1989]. Sidebands at approximately plus and minus $500 \mathrm{~Hz}$ of the carrier frequency (11.9 and $12.65 \mathrm{kHz}$ ) of Alpha pulses have been observed in the ionosphere by the AUREOL 3 satellite [Tanaka et al., 1987; Lagoutte et al., 1989]. They are reduced in amplitude by about $20 \mathrm{~dB}$ with respect to the carrier. They have been shown to exhibit phase relationships with the transmitted

${ }^{1}$ On leave from Space Research Institute (IKI), Moscow.

${ }^{2}$ On leave from Geophysical Institute, Prague.

Copyright 1991 by the American Geophysical Union.

Paper number 91JA00695. 0148-0227/91/91JA-00695\$05.00 signal and a natural emission around $500 \mathrm{~Hz}$, just above the local proton gyrofrequency.

In order to explain the 55-Hz sidebands, Bell [1985] invoked a nonlinear interaction between gyroresonant energetic electrons and coherent waves in the equatorial regions. The interaction does not need to be local, i.e., close to the observation region. No generation model is given by Tanaka et al. [1987] and Lagoutte et al. [1989] for the $500-\mathrm{Hz}$ sidebands. According to the phase relationships mentioned above and to the apparent electrostatic nature of the signal, it is thought that the sidebands are produced by a three-wave nonlinear interaction at the vicinity of the satellite. At first sight the $500-\mathrm{Hz}$ sidebands observed on AUREOL 3 seem to correspond to the Riggin and Kelley (1982) prediction in which the transmitted wave decays into a lower hybrid wave and an ion-acoustic type of oscillation. To account for the existence of two symmetric spectral peaks, one may replace the three-wave parametric instability considered by these authors by a four-wave parametric instability (or modulational instability) as suggested by Lee and Kuo [1984] and Groves et al. [1988]. According to this scheme, the ELF branch is due to a purely growing (zero frequency) electrostatic mode with wave vectors $k$ large enough to provide sidebands at $\pm|k . V$,$| off the transmitter$ frequency $\left(V_{s}\right.$ is the satellite velocity). This mode is excited in course of a four-wave process by the incident VLF transmitter wave. In our case the ELF branch is clearly electromagnetic and as such is of natural origin. Therefore another explanation in accord with our experimental data has to be found.

Electromagnetic band-limited ELF emissions, with a lower cutoff frequency near the local proton gyrofrequency, are currently observed on low-altitude satellites [Gurnett and Burns, 1968; Mosier, 1971; Muzzio and Angerami, 1972; Rauch et al., 1985; Hayakawa et al., 1990]. However, enhancements in the amplitude of those emissions at several transmitter longitudes [Parrot, 1990] do not enable us to completely rule out the possibility of a contribution of the transmitted waves in the production of ELF emissions.

The aim of the present paper is to propose another mechanism for the production of the $500-\mathrm{Hz}$ sidebands. It is based on a nonlinear coupling between the transmitted wave 
and the ELF emission above the local proton gyrofrequency. The sidebands are shown to be forced oscillations, excited only where the coupling takes place. An estimation of the amplitude of the sideband signals is given.

The organization of the paper is as follows. Section 2 is devoted to the AUREOL 3 data. The TBF ONCH experiment measuring the waves in the frequency range $10 \mathrm{~Hz}$ to $16 \mathrm{kHz}$ is described. The $500-\mathrm{Hz}$ sidebands already analyzed by Tanake et al. [1987] and Lagoutte et al. [1989] are reexamined. The absolute values of the power spectra, which could not be produced in the previous papers, are estimated using outputs of filter bank data. In section 3 a nonlinear coupling model is proposed and the amplitudes of the sideband signals are compared with the measured data. Finally, section 4 offers provisional conelitsions.

\section{The DATA}

AUREOL 3 is a three axis stabilized-satellite launched on September 21, 1981, on a quasi-polar orbit (apogee: $2012 \mathrm{~km}$, perigee: $408 \mathrm{~km}$, inclination: $82^{\circ} 5$, period: $109.5 \mathrm{~min}$ ). The set of experiments aboard the satellite constituted the socalled ARCAD 3 project.

The TBF ONCH experiment [Berthelier et al., 1982] measured two electric and three magnetic wave field components in the ELF/VLF frequency range. For technical reasons the measurements were not performed in Cartesian reference systems. One electric component $\left(E_{z}\right)$ was measured by means of two spheres located at the end of an insulated boom parallel to the vertical $Z$ axis of the spacecraft. The distance between the two spheres is $2.36 \mathrm{~m}$. The $Z$ axis is approximately along the vertical. The second electric component $\left(E_{H}\right)$ was measured by means of two spheres located on two different booms. The distance between the spheres is $7.43 \mathrm{~m}$. The $H$ direction is $11^{\circ} 5$ off the plane perpendicular to the $Z$ axis. As far as the magnetic components are concerned, $B_{Z}$ is taken along $Z$. $B_{X}$ is in the direction of the average velocity vector and in a plane perpendicular to $B_{\mathrm{Z}} . B X_{45}$ is in the same plane and makes an angle of $45^{\circ}$ with $B_{X}$.

Three types of data were transmitted to the ground. The data of the first type ( $\mathrm{Cl}$ mode) are the waveforms of the five electromagnetic components simultaneously recorded in the frequency band $10-1500 \mathrm{~Hz}$. They require direct telemetry, which means that they are recorded when the satellite is in view of a telemetry station. They allow detailed study of ELF emissions particularly around the proton gyrofrequency. The data of the second type ( $\mathrm{C} 2$ mode) are waveforms in the frequency band $70 \mathrm{~Hz}-16 \mathrm{kHz}$ associated with a unique electromagnetic component. One has either a fixed component, or four of them are in a cyclic sequence $\left(E_{Z}\right.$, $\left.B X_{45}, E_{H}, B_{X}\right)$. The component is changed each $4 \mathrm{~s}$. As the C2 mode data also require direct telemetry they are obtained over time intervals where the $\mathrm{Cl}$ mode data are not collected. They are mainly devoted to morphological studies and more specifically to the observation of the phenomena induced by the ground-based VLF transmitters. According to the uncertainties in the transfer functions of all the on-board systems the $\mathrm{C} 2$ mode data are not computed in physical units. The data of the third type are filter bank data recorded nearly continuously according to different modes of functioning. The center frequencies and bandwidths are given by Berthelier et al. [1982]. They are mainly used for general surveys. We will use them here to calibrate the $\mathrm{C} 2$ mode data.

Sidebands have been identified on $\mathrm{E}_{\mathrm{H}}$ autopower spectra derived from $\mathrm{C} 2$ mode data. The observations were made on two different orbits, during short time intervals (approximately $40 \mathrm{~s}$, i.e., $320 \mathrm{~km}$ ), slightly southward of the Komsomolskon-Amur Alpha station $\left(50^{\circ} 5 \mathrm{~N}, 135^{\circ} \mathrm{E}\right)$, at altitudes around $1200 \mathrm{~km}$ and $L$ values between 1.7 and 2 (see Figure 1 of
Tanaka et al. [1987] An example is presented in Figure 1. The $B X_{45}$ power spectrum (Figure $1 b$ ), computed $4 \mathrm{~s}$ before the $E_{H}$ power spectrum (Figure $1 a$ ), is practically identical to the $B_{X}$ power spectrum calculated $4 \mathrm{~s}$ after $E_{H}$. The integration times have been chosen in such a way that they cover the time duration of the transmitted pulses. The frequency resolution is $50 \mathrm{~Hz}$. Figure 1 a clearly identifies the transmitter frequency $(12.65 \mathrm{kHz})$, the ELF emission with a peak at $500 \mathrm{~Hz}$, i.e., just at or above the local gyrofrequency (464 $\mathrm{Hz}$ but beween 450 and $500 \mathrm{~Hz}$ according to our frequency resolution), and an upper sideband at $500 \mathrm{~Hz}$, with a slightly lower signal to noise ratio. Figure $1 b$ is corrupted by spectral lines (spaced by $1.6 \mathrm{kHz}$ ) due to the spacecraft subsystems. However, one observes that the ELF emission just above the local gyrofrequency (practically the same as in Figure 1a) and the emission at the transmitter frequency are electromagnetic whereas the sidebands are electrostatic. The power spectra of the ELF emission on $E_{H}$ and $B X_{45}$ are consistent with those observed at other longitudes [Rauch et al., 1985; Hayakawa et al., 1990], with an unexplained attenuation band above the lower frequency peak.

Statistics over four set of observations, made on August 11, 1982, have been carried out. The transmitter frequency is at $11.90 \mathrm{kHz}$ for two sets of observations and at $12.65 \mathrm{kHz}$ for the two others. Histograms of the frequency of the $E H$ power spectra maxima are represented in Figure 2 with frequency bins of $500 \mathrm{~Hz}$. The ELF emissions have their maxima between 500 and $600 \mathrm{~Hz}$, i.e., just above the local proton gyrofrequency. The maxima of the lower sidebands are 500 to $600 \mathrm{~Hz}$ below the transmitter frequency. The maxima of the upper sidebands, slightly more dispersed in frequency, are 500 to $650 \mathrm{~Hz}$ above the transmitter frequency.

As already mentioned, an electromagnetic ELF band with a lower frequency cutoff near the local proton gyrofrequency is currently observed on low-altitude satellites [Gurnett and Burns, 1968; Mosier, 1971; Muzzio and Angerami, 1972; Rauch et al., 1985; Hayakawa et al., 1990]. As first suggested by Mosier [1971], then experimentally pointed out by F. Lefeuvre et al. (Propagation characteristics of low-altitude dayside ELF emissions, submitted to Journal of Geophysical Research 1991), ELF waves, generated at high altitude in diffusive aurora regions, may propagate across the plasmapause to lower latitudes. The lower frequency part, refracted upward at the two-ions cutoff frequency, is trapped within the plasmasphere.

Let us return to the data of Figure 1. Because filter banks and $\mathrm{C} 2$ mode are both connected to the $E_{H}$ measurements, one can calibrate the autopower spectrum of $E_{H}$ by comparing the output of a low-frequency wideband filter (here a filter centered around $725 \mathrm{~Hz}$ ) with the integral of the power spectrum in the same frequency band. As a result we obtain values of the order of $50 \mu \mathrm{V} / \mathrm{m} / \sqrt{\mathrm{Hz}}$ for the band around $500 \mathrm{~Hz}$. Because peak at $12.65 \mathrm{kHz}$ is approximately $6 \mathrm{~dB}$ above the peak at $500 \mathrm{~Hz}$, we have about $100 \mu \mathrm{V} / \mathrm{m} / \sqrt{\mathrm{Hz}}$ for the transmitted frequency. The transfer function of the VLF filter is nearly flat in the frequency domain we are interested in and does not introduce inaccuracies greater than $15 \%$. The value obtained for the transmitter amplitude is consistent with values directly obtained over the Novosibirsk Alpha station and with estimations made from the radiating power of the transmitter.

As the $B X_{45}$ measurements are not connected to the filter banks, one has no direct way of calibrating the $B X_{45}$ autopower spectra of Figure $1 b$. However, statistics made on the C1 mode at longitudes close to the one of the Komsomolsk-on-Amur station have shown that, at frequencies just above the local proton gyrofrequency, the $c B X_{45} / E_{H}$ ratio, with $c$ the velocity of light, was of the order of 60 . Assuming that the statistical properties of the ELF emission are the same at the time intervals corresponding to Figures $1 a$ and 
a

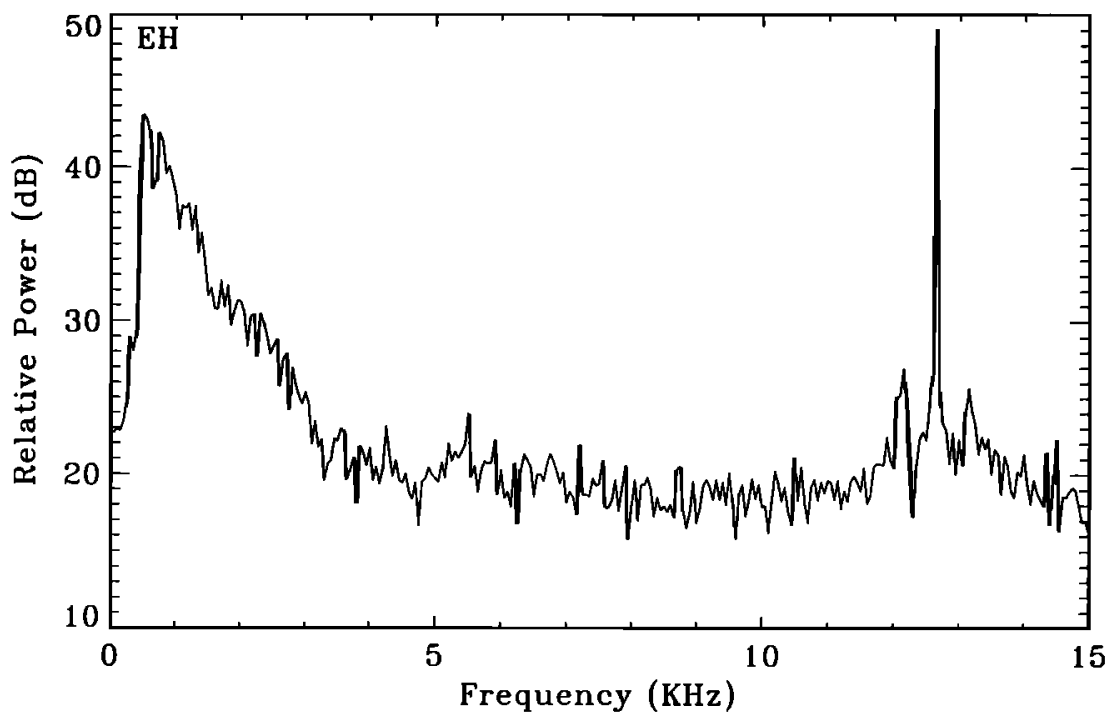

b

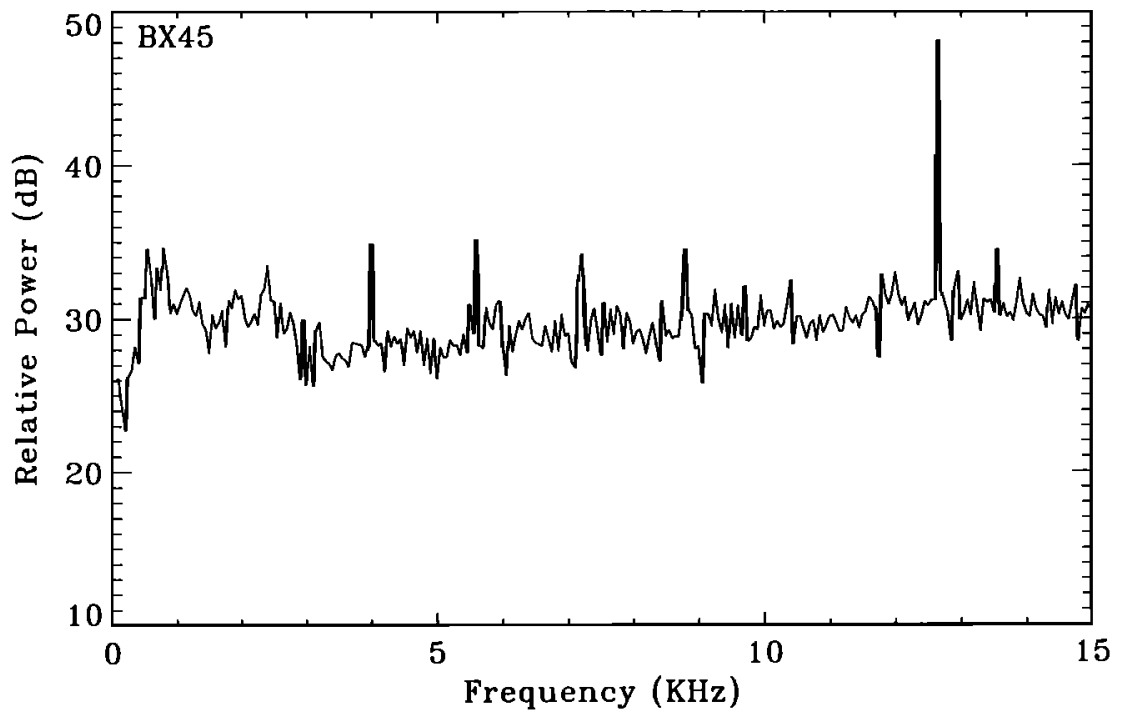

Fig. 1. Averaged power spectral densities of the (a) $E_{H}$ and (b) $B X_{45}$ wave field components above the Konsomolsk-on-Amur Alpha station on August 111982 , around $1190 \mathrm{~km}$ altitude, $1310 \mathrm{MLT}, 135^{\circ} 7$ geographic longitude, and $43^{\circ}$ Invariant latitude.

$1 b$, we obtain $7 \mathrm{~m} \gamma / \sqrt{\mathrm{Hz}}$ around $500 \mathrm{~Hz}$. This leads to 30 $\mathrm{m} \gamma / \mathrm{VHz}$ at the frequency of the transmitter.

From the estimation of the electric and magnetic power at $12.65 \mathrm{kHz}$, one may evaluate the values of the refractive index of the transmitted wave. From Maxwell's equation we have $n \geq C B / E=100$. Taking the whistler mode approximation of the refractive index [Helliwell, 1965] and assuming the plane-wave approximation, we find that the $\theta_{1}$ angle made of the $k$ vector with the Earth's magnetic-field direction $B_{0}$ must be greater than $87^{\circ}-88^{\circ}$. The exact value depends on the value we take for the ratio of the electron-plasma frequency to the electron gyrofrequency. In the region of the ionosphere considered here, it varies from 1.2 to 1.5 .

As regards the $\theta_{2}$ angle taken by the ELF emissions, estimations are slightly more complex. First, the ionic composition must be taken into account. Second, as deduced from the $\mathrm{Cl}$ mode data recorded at the longitude of the Komsomolsk-on-Amur station, the plane-wave hypothesis is generally not valid. Taking $n \geq c B X_{45} / E_{H}$ and considering
$50 \%$ of $\mathrm{H}^{+}$ions and $50 \%$ of $\mathrm{O}^{+}$ions, one gets $\theta_{2} \geq 30^{\circ}$. This value increases with the $\mathrm{H}^{+}$concentration and becomes $\theta_{2} \geq 40^{\circ}$ for $90 \%$ of $\mathrm{H}^{+}$ions. Now, wave analysis made on Cl mode data have shown that the Means [1972] method generally gives $\theta_{2} \approx 65^{\circ}\left( \pm 5^{\circ}\right.$ ) for ELF emission recorded just above the local proton gyrofrequency at low $L$ values ( $\mathrm{L} \leq 2)$. Applications of a two-plane wave model [Hayakawa et al., 1990] indicate that the maximum of energy is conveyed by $\theta_{2}$ values larger than $65^{\circ}$. For this reason, we have taken $\theta_{2} \geq 65^{\circ}$.

\section{Nonlinear Coupling Model}

According to the experimental data presented above, we are led to explain the sidebands as a result of nonlinear coupling of the VLF transmitter wave and the natural ELF emission. The VLF wave $\mathbf{E}_{1}(\mathbf{r}, t)=\mathbf{E}_{1} e^{i k r-i \omega t}+$ c.c. propagates through the ionosphere as a whistler mode 

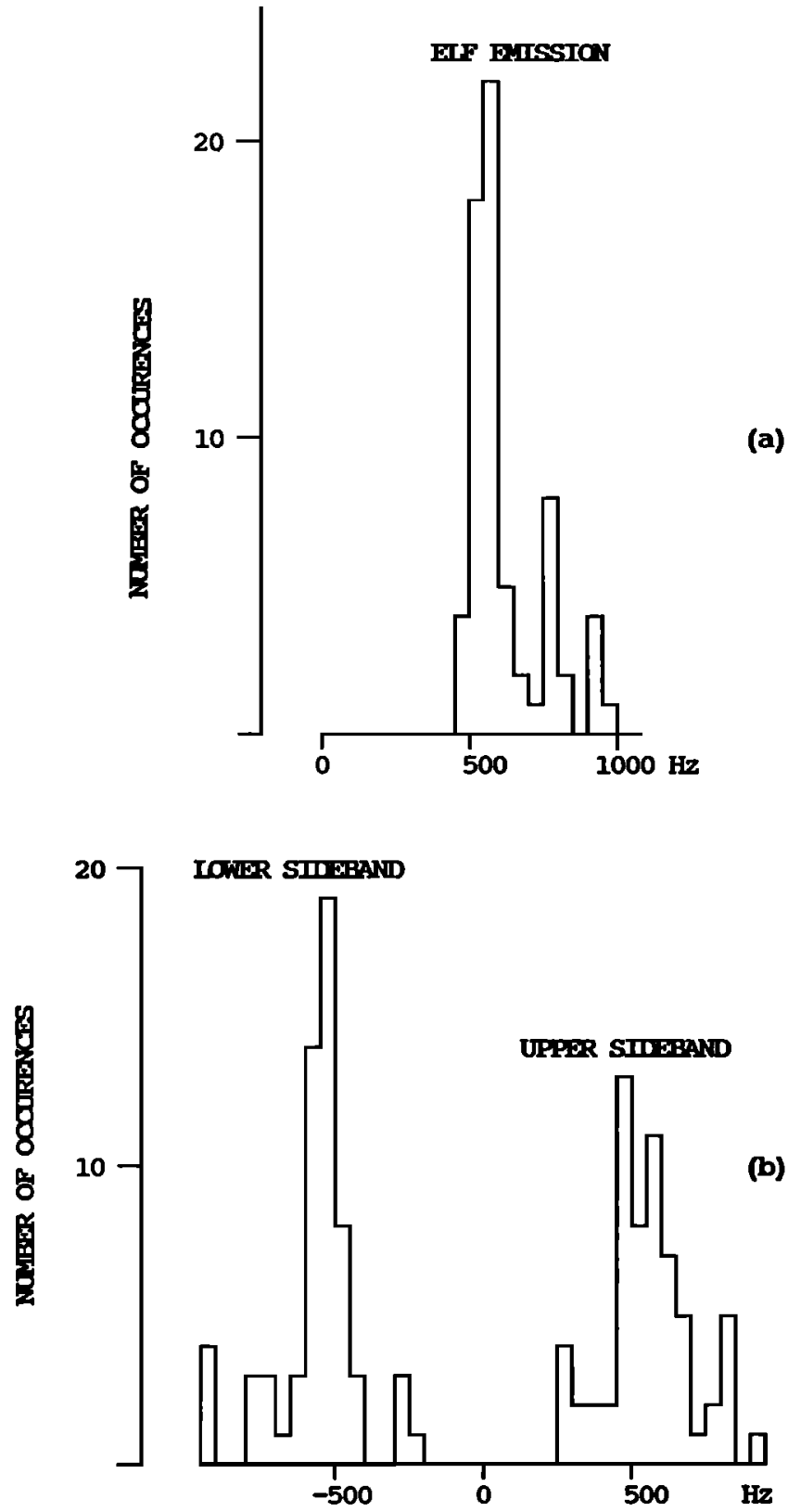

Fig. 2. Distribution in frequency of the power spectra maxima for the (a) ELF emission and the $(b)$ sidebands for four sets of observations made on August 11, 1982 (see text).

$$
\omega_{k_{1}}=\omega_{H} k_{12} / k_{1}\left(1+\omega_{p}^{2} / k_{1}^{2} c^{2}\right)^{-1}
$$

For the transmitted frequency $\omega_{1} / 2 \pi \approx 12 \mathrm{kHz}$, the corresponding wave number is estimated to be $k^{1} \approx 2.10^{-4} \mathrm{~cm}^{-1}$. Taking the corresponding plasma frequency and the electron gyrofrequency, we can see that the whistler propagates with $\omega_{p}{ }^{2} / k_{1}{ }^{2} c^{2} \approx 1$ at a large angle $\theta$ to the magnetic field.

The characteristic frequency $\omega_{k}$ of the ELF wave is slightly above the ion gyrofrequency, and its wave vector is estimated at $k_{2} \approx 3.10^{-5} \mathrm{~cm}^{-1}$. These waves generally propagate at a large angle to the geomagnetic field. As $\omega_{p}^{2} / k_{2}{ }^{2} c^{2}>1$, it is described by

$$
\omega_{k_{2}} \approx \omega_{H} k_{2 z} / k_{2}\left(k_{2}^{2} c^{2} / \omega_{p}^{2}\right)
$$

The nonlinear coupling of the modes (2) and (3) causes the beat-wave field at the combination frequencies (the sidebands),

$$
\omega_{ \pm}=\omega_{k_{1}} \pm \omega_{k_{2}}
$$

The resulting sideband wave numbers are matched according to

$$
k_{ \pm}=k_{1} \pm k_{2}
$$

It should be pointed out that the eigenmode frequency matching cannot be met in this case,

$$
\omega_{k_{1}} \pm k_{2} \neq \omega_{k_{1}} \pm \omega_{k_{2}}
$$

In other words, the sidebands are not plasma eigenmodes but forced oscillations excited only where the VLF to ELF wave coupling takes place.

Consequently, the linear permitivity function $\varepsilon_{L}\left(\omega_{ \pm}, k_{ \pm}\right)$ is not equal to zero for the sidebands modes. In this case the electric field of the sidebands, $E_{ \pm}$, may be schematically written as

$$
E_{ \pm}=\frac{\varepsilon^{(2)}\left(\omega_{k_{1}}, \omega_{k_{2}}, k_{1}, k_{2}\right)}{\varepsilon_{L}\left(\omega_{ \pm}, k_{ \pm}\right)} E_{1} E_{2}
$$

with $E_{1}, E_{2}$ respectively the VLF and ELF wave amplitudes and $\varepsilon^{(2)}$ the second-order (nonlinear) dielectric function.

In case of sufficiently strong interacting VLF and ELF waves the resulting beats may be observed in the experiments as the sidebands shown above. A similar process of nonlinear coupling of high-frequency resonance-cone modes with lowfrequency ion sound waves, resulting in excitation of whistlers in that case, was considered by Fiala et al. [1987].

Thereafter, we show how to obtain the equation of type (6) in our case. A set of three equations $(i=1,2,3)$ for the sideband electric field components $E_{i \pm}$, the wave equation, may be written as

$$
\sum_{j=1}^{3} \lambda_{i j} E_{j \pm}=\frac{4 \pi i}{c^{2}} \omega_{ \pm} j_{i \pm}^{N}
$$

and the $\lambda_{i j}$ tensor components in Cartesian coordinates $(x, y$, 2), 2 axis along the magnetic field, are

$$
\begin{aligned}
& \lambda_{i j}=k^{2}-k_{\mathrm{i}}^{2}-\frac{\omega^{2}}{c^{2}} \varepsilon_{i i}, i=x, y, z, i=j \\
& \lambda_{i j}=-k_{\mathrm{i}} k_{\mathrm{j}}-\frac{\omega^{2}}{c^{2}} \varepsilon_{i j}, i j=x, y, z, i \neq j
\end{aligned}
$$

and the cold plasma dielectric tensor is

$$
\begin{gathered}
\varepsilon_{z x}=\varepsilon_{y y}=1-\omega_{p}^{2} /\left(\omega^{2}-\omega_{H}^{2}\right) \\
\varepsilon_{z z}=1-\omega_{p}^{2} / \omega^{2} \\
\varepsilon_{x y}=-\varepsilon_{x y}=-i \omega_{p}^{2} \omega_{H} /\left(\omega\left(\omega^{2}-\omega_{H}^{2}\right)\right)
\end{gathered}
$$

with the remaining components equal to zero. Everywhere in (8) and (9) the wave number is that of the sidebands as well as the frequency $\left(\omega \rightarrow \omega_{ \pm}, k \rightarrow k_{ \pm}\right)$.

The source term on the right-hand side in (7) is the nonlinear current resulting from the interaction between the VLF and ELF modes. 
Since the wave component perpendicular to the magnetic field $E_{\perp}$ is measured in the experiment, we write the solution of (8) in the form $\left(E_{x}=E_{\perp} \cos \chi, E_{y}=E_{\perp} \sin \chi\right)$ :

$$
\begin{gathered}
k_{\perp} E_{\perp \pm}=\frac{4 \pi i \omega}{\omega_{\mathrm{p}}^{2}\left(1+\omega_{\mathrm{p}}^{2} / \mathrm{k}^{2} c^{2}\right)} \frac{1}{\varepsilon_{\mathrm{L}}(\omega, \mathrm{k})} \\
\left.\left.\left\{\mathbf{k}_{\perp}\right]_{\perp}^{N}+i \frac{\omega_{p}^{2}}{k^{2} c^{2}} \frac{\omega}{\omega_{H}}\left[k_{J}\right\rfloor^{N}\right]_{z}+\frac{k_{z} j_{z}^{N}}{1+\omega_{p}^{2} / k^{2} c^{2}}\right\}
\end{gathered}
$$

with

$$
\varepsilon_{L}(\omega, k)=k_{2}^{2} /\left(k^{2}\left(1+\omega_{p}^{2} / k^{2} c^{2}\right)^{2}\right)-\omega^{2} / \omega_{H}^{2}
$$

We remind once more that (10) represents in effect two equations, for lower $\left(\omega_{.}, k_{-}\right)$and upper $\left(\omega_{+}, k_{+}\right)$sidebands. Now the nonlinear current densities $j^{N}\left(\omega_{ \pm}, k_{ \pm}\right)$are to be determined. To do this, we use kinetic approach and we solve the kinetic equation for the electron distribution function by successive approximations.

The perturbation of the distribution function is developed from a series in wave amplitudes

$$
f_{k}=f_{k}^{(1)}+f_{k}^{(2)}+\ldots
$$

of which only the two terms (linear and quadratic) are needed. The nonlinear current is then given by $(e=-|e|)$

$$
\int_{k_{ \pm}}^{N}=e \int v f_{k_{ \pm}}^{(2)} d v
$$

We have relegated the details of this calculation to the appendix, where it is shown that only the component $j_{z k}$ along the magnetic field is responsible for interaction

$$
j_{2 k+}^{N}=\frac{i}{2 \pi} \frac{e}{m} \frac{\omega_{p}^{2}}{\omega_{H} \omega_{+}} \frac{k_{12}}{\omega_{k_{1}}} E_{\perp k_{1}} E_{\perp k_{2}} \sin \left(\chi_{1}-\chi_{2}\right)
$$

As there remains now only the last term on the right-hand side of (10), we obtain for the sideband field the following expression:

$$
\mathrm{E}_{\perp+}=\frac{e}{m} \frac{k_{1}}{\omega_{H}} \frac{E_{\perp k_{1}} E_{\perp k_{2}}}{\delta \omega_{+}} \sin \left(\chi_{1}-\chi_{2}\right)
$$

For the lower sideband $E_{\perp-}$ the $E_{\perp k_{2}}$ is to be replaced by its complex conjugate, $\delta \omega_{+}$by $-\delta \omega_{\text {. }}$ and

$$
\delta \omega_{ \pm}=\omega_{ \pm}-\omega_{H} \frac{k_{2 \pm} k_{ \pm}}{k_{ \pm}^{2}+\omega_{p}^{2} / c^{2}}
$$

In our case $\left(k_{1}>k_{2}, \omega_{1}>\omega_{2}, \omega_{p}{ }^{2} / k_{1}{ }^{2} c^{2} \leq 1\right)$ it could be shown that $\delta \omega_{+} \approx \delta \omega_{-} \approx \omega_{2}$, and we propose the following estimate of the sideband amplitude normalized to the transmitter wave electric field

$$
E_{ \pm} / E_{1} \approx \frac{e E_{2}}{m \omega_{H}} \frac{1}{\lambda_{1} \delta f}=\frac{E_{2}}{B_{0}} \frac{1}{\lambda_{1} \delta f}
$$

with $\lambda_{1}[m]$ the transmitter wavelength and $\delta f, E_{2}[\mathrm{~V} / \mathrm{m}]$ the ELF wave frequency and amplitude respectively and $B_{0}$ (in tesla) the Earth's magnetic field.

\section{Conclusion}

We have shown that a nonlinear coupling between VLF and ELF waves gives rise naturally to sidebands, whose amplitude is given by (15). The frequency mismatch $\delta \omega_{ \pm}$is a quite complicated function of the wavelengths and direction of propagation of the interacting modes. The dispersion relations (1) and (2), and the requirement that the sidebands be equal,

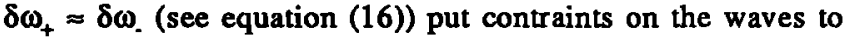
interact effectively. In the observed case the sideband to transmitter wave-amplitude ratio $E_{ \pm} / E_{1}$ is about $5 \times 10^{-2}$. It can be readily shown that the right-hand side of (17) is about $10^{-2}$ in case $k_{1}>k_{2}, \omega_{1}>\omega_{2}, \omega_{p}{ }^{2} / k_{1}{ }^{2} c^{2}<1$, and for waves propagating at comparable angles to the magnetic field $\left(\theta_{1}-\theta_{2}\right)$. We took the low-frequency band electric field $E_{2}$ to be of several millivolts per meter as indicated by the data of Figure 1 and the Earth's magnetic field of $3 \times 10^{-5}$ esla $\left(f_{H}-850 \mathrm{kHz}\right)$.

In that case the mismatch frequency $\delta \omega_{+} \approx \delta \omega_{.} \leq \omega_{2}$ and for $\lambda_{1}$ of the order of $100 \mathrm{~m}$ we find a satisfactory value of the sideband amplitude.

In other cases $\left(\theta_{1}\right.$ very different from $\left.\theta_{2}\right)$ the sideband amplitude is about an order of magnitude lower. This may explain the fact that sidebands are not observed very often. In this respect it is even more difficult to estimate the width of sidebands above the noise level. It should be noted that there is no threshold involved in our mechanism. The appearing width is then given by the rapidity in the decrease of the ELF spectrum as well as by the resolution of the receiver.

Then, we conclude that the sidebands are satisfactorily explained by the coupling of ELF to VLF waves and that the observed amplitude is determined by a selective process in the interacting wave vectors.

\section{APPENDIX}

To obtain the nonlinear current which appears in the source term of (7), we chose a kinetic approach. The perturbation of the electron distribution function $f_{0}\left(f_{0}\right.$ is supposed to be an isotropic Maxwellian in final formulas) is developed in a series of wave amplitudes up to the second (quadratic) term,

$$
f_{k}=f_{k}^{(1)}(E)+f_{k}^{(2)}\left(E^{2}\right)
$$

Another scheme of successive approximations is needed to solve the problem. For this reason we use the fact that $k r_{H}$ ( $r_{H}$ being the electron Larmor radius), is a small parameter in our problem. Each of the terms on the right-hand side of (A1) is written as a power series in $k r_{H}$, and we shall see that the first two terms in each of them will be needed.

Consequently, we can write

$$
f_{k}=f_{0 k}^{(1)}+f_{l k}^{(1)}+f_{0 k}^{(2)}+f_{l k}^{(2)}
$$

with a first-order differential equation for each of the terms $\left(\omega^{2} / \omega_{H}^{2}>k^{2} r_{H}^{2}\right.$ is used hereafter):

$$
\frac{\partial f_{\partial k}^{(1)}}{\partial \alpha}+i \frac{\omega_{k}}{\omega_{H}} f_{o k}^{(1)}=\sigma_{0}^{(1)}(\alpha)
$$

with

$$
\sigma_{0}^{(1)}(\alpha)=\frac{\mathrm{e}}{m \omega_{H e}}\left\{E_{k \perp} \cos (\alpha-\chi) \frac{\partial f_{0}}{\partial v_{\perp}}+E_{k z} \frac{\partial f_{0}}{\partial v_{\mathrm{z}}}\right\}
$$

and 


$$
\begin{gathered}
\frac{\partial f_{j k}^{(1)}}{\partial \alpha}+\mathrm{i} \frac{\omega_{k}}{\omega_{\mathrm{H}}} f_{1 k}^{(1)}=i \frac{k_{\perp} \mathbf{v}_{\perp}}{\omega_{\mathrm{H}}} \cos (\alpha-\phi) f_{\mathrm{Ok}}^{(1)} \\
-i \frac{k_{z} \mathbf{v}_{z}}{\omega_{H}} f_{0 k}^{(1)}+\sigma_{1 k}^{(1)}(\alpha)
\end{gathered}
$$

with

$$
\begin{gathered}
\sigma_{1 k}^{(1)}=\frac{e}{m \omega_{H e}}\left[E_{z k} \frac{k_{\perp}}{\omega_{k}} \cos (\alpha-\phi)-E_{\perp k} \frac{k_{z}}{\omega_{k}} \cos (\alpha-\chi)\right] \\
\cdot\left(v_{z} \frac{\partial f_{0}}{v_{\perp}}-v_{\perp} \frac{\partial f_{0}}{\partial v_{z}}\right)
\end{gathered}
$$

Note that $\sigma_{1 k}^{(1)}=0$ for an isotropic Maxwellian distribution $f_{0}$. Now

$$
\frac{\partial f_{0 k_{+}}^{(2)}}{\partial \alpha}+i \frac{\omega_{+}}{\omega_{H}} f_{0 k_{+}}^{(2)}=\sigma_{0 k_{+}}^{(2)}
$$

with

$$
\begin{aligned}
& \sigma_{0 k_{+}}^{(2)}(\alpha)=\frac{e}{m \omega_{H}}\left\{E_{\perp k_{2}} \cos \left(\alpha-\chi_{k_{2}}\right) \frac{\partial f_{0 k_{1}}^{(1)}}{\partial v_{\perp}}+E_{z k_{2}} \frac{\partial f_{0 k_{1}}^{(1)}}{\partial v_{2}}\right. \\
& \left.+E_{\perp k_{2}} \sin \left(\chi_{k_{2}}-\alpha\right) \frac{1}{v_{\perp}} \frac{\partial f_{0 k_{1}}^{(1)}}{\partial \alpha}\right\}+\left(\begin{array}{l}
k_{1} \rightarrow k_{2} \\
k_{2} \rightarrow k_{1}
\end{array}\right)
\end{aligned}
$$

where the last term in parenthesis stands for the preceding one with indices changed as indicated. The corresponding equation for the lower sideband $\left(\omega, k_{-}\right)$is obtained by changing $\omega_{+}$to $\omega_{-}, k_{+}$to $k_{-}$and $E_{\perp k_{2}}$ to $E_{\perp k_{2}}^{*}$ (complex conjugate). The last term obeys

$$
\begin{gathered}
\frac{\partial f_{1 k_{+}}^{(2)}}{\partial \alpha}+\mathrm{i} \frac{\omega_{+}}{\omega_{H}} f_{1 k_{+}}^{(2)}=i \frac{k_{\perp+} v_{\perp}}{\omega_{H}} \cos \left(\alpha-\phi_{k_{+}}\right) f_{0 k_{+}}^{(2)} \\
-i \frac{k_{2+} v_{2}}{\omega_{H}} f_{0 k_{+}}^{(2)}+\sigma_{1 k_{+}}^{(2)}(\alpha)
\end{gathered}
$$

with

$$
\begin{gathered}
\sigma_{1 k_{+}}^{(2)}(\alpha)=\frac{e}{m \omega_{H}}\left\{E _ { \perp k _ { 2 } } \left[\cos \left(\alpha-\chi_{k_{2}}\right) \frac{\partial f_{1 k_{1}}^{(1)}}{\partial v_{\perp}}\right.\right. \\
\left.+\sin \left(\chi_{k_{2}}-\alpha\right) \frac{1}{v_{\perp}} \frac{\partial f_{0 k_{1}}^{(1)}}{\partial \alpha}\right]+E_{z k_{2}} \frac{\partial f_{1 k_{1}}^{(1)}}{\partial v_{z}} \\
+\left(E_{z k_{2}} \frac{k_{2 \perp}}{\omega_{k_{2}}} \cos \left(\alpha-\phi_{k_{2}}\right)-E_{\perp k_{2}} \frac{k_{2 z}}{\omega_{k_{2}}} \cos \left(\alpha-\chi_{k_{2}}\right)\right) \\
\cdot\left(v_{z} \frac{\partial f_{0 k_{1}}^{(1)}}{\partial v_{\perp}}-v_{\perp} \frac{\partial f_{0 k_{1}}^{(1)}}{\partial v_{z}}\right) \\
+\left[E_{2 k_{2}} \frac{k_{2 \perp}}{\omega_{k_{2}}} \sin \left(\phi_{k_{2}}-\alpha\right)-E_{\perp k_{2}} \frac{k_{2 z}}{\omega_{k_{2}}} \sin \left(\chi_{2}-\alpha\right)\right] \frac{v_{z}}{v_{\perp}} \frac{\partial f_{0 k_{1}}^{(1)}}{\partial \alpha}
\end{gathered}
$$

$$
\left.+E_{z k_{2}} \frac{k_{21}}{\omega_{k_{2}}} \sin \left(\phi_{k_{2}}-\alpha\right) \frac{\partial f_{0 k_{1}}^{(1)}}{\partial \alpha}\right)+\left(\begin{array}{l}
k_{1} \rightarrow k_{2} \\
k_{2} \rightarrow k_{1}
\end{array}\right)
$$

The intervening angles are those that the corresponding vectors perpendicular to the magnetic field make with the $x$ axis ( $\alpha$ for $v_{\perp}, \phi$ for $k_{\perp}$ and $\chi$ for $E_{\perp}$ ). The first moment of $f_{k}$ gives the current density

$$
\mathbf{j}_{k}=e \int d \mathbf{v} \mathbf{v} f_{k}
$$

Now the solution to (A3) is

$f_{0 k}^{(1)}=\frac{e}{m \omega_{H}}\left\{E_{\perp k} \frac{\partial f_{0}}{\partial v_{\perp}}\left[\sin \left(\alpha-\chi_{k}\right)+i \frac{\omega_{k}}{\omega_{H}} \cos \left(\alpha-\chi_{k}\right)\right]\right.$

$$
-\mathrm{i} \frac{\omega_{H}}{\omega_{k}} E_{z k} \frac{\partial f_{0}}{\partial \mathrm{v}_{z}} \text { ] }
$$

The current corresponding to $f_{0 k}^{(1)}$ is already included in the cold plasma dielectric tensor equation (9).

We do not write down the lengthy expressions for the remaining terms of the approximation (A2). But we note that the term $f_{0 k}^{(2)}$ does not contribute to the nonlinear current,

$$
e \int v f_{\mathrm{O}_{+}}^{(2)} d v=0
$$

which is the reason for keeping the next term in the series. The nonlinear current appearing on the right-hand side of (7) is obtained as

$$
j_{ \pm}^{N}=e \int \mathrm{v} f_{1 k_{ \pm}}^{(2)} d v
$$

The part of $f_{1 k}^{(2)}$ that does not depend on $\alpha$ contributes to the parallel current $j_{2}^{N}$ and is given by (14). The part that depends on $\alpha$ results in a perpendicular current $j_{\perp}^{N}$. This is found to be small when $\omega_{ \pm}^{2} / \omega_{H}{ }^{2}<1$ and $\omega_{p}{ }^{2} / k_{+}{ }^{2} c^{2} \leq 1$. This has been expected as the sidebands are very nearly potential waves, and for that reason we keep only the most important term with $j_{2}^{N}$ in (10).

Acknowledgments. The authors wish to thank J. J. Berthelier, P. I. of the TBF experiment, for providing us with the data, and their colleague $M$. Parrot for his help in analyzing the filter bank data. The ARCAD 3 project launched on board the AUREOL 3 satellite was jointly supported by INTERCOSMOS (URSS) and CNES (France).

The Editor thanks the two referees for assistance in evaluating this paper.

\section{REFERENCES}

Bell, T. F., High amplitude VLF transmitter signals and associated sidebands observed near the magnetic equatorial plane on the ISEE 1 satellite, J. Geophys. Res., 90, 2792, 1985.

Bell, T. F., and H. D. Ngo, Electrostatic waves stimulated by coherent VLF signals propagating in and near the inner radiation belt, J. Geophys. Res., 93, 2599, 1988.

Bell, T. F., and H. D. Ngo, Electrostatic lower hybrid waves excited by electromagnetic whistler mode waves scattering from planar magnetic-field-aligned plasma density irregularities, J. Geophys. Res., 95, 149, 1990.

Bell, T. F., H. G. James, U. S. Inan, and J. P. Katsufrakis, The apparent spectral broadening of VLF transmitter signals during transionospheric propagation, J. Geophys. Res., 88, 4813, 1983. 
Berthelier, J. J., F. Lefeuvre, M. M. Mogilevsky, O. A. Molchanov, Yu. I. Galperin, J. F. Karczewski, R. Ney, G. Gogly, C. Guerin, M. Levêque, J. M. Moreau, and F. X. Sené, Measurements of the VLF electric and magnetic components of waves and DC electric field on board the AUREOL 3 satellite: The TBF-ONCH experiment, Ann. Geophys., 38, 643, 1982.

Chmyrev, V. M., M. M. Mogilevsky, O. A. Molchanov, Y. P. Sobolev, E. E. Titova, T. A. Yakhina, R. N. Suncheleev, V. A. Gladyshev, N. V. Baranets, N. V. Dzhordzhio, Y. I. Galperin, and A. V. Streltsov, Parametric excitation of ELF waves and acceleration of ions during the injection of strong VLF waves into the ionosphere, Kosm. Issled., 27, 249, 1989.

Fiala, V., E. N. Kruchina, and V. I. Sotnikov, Whistler excitation by transformation of lower oblique resonance waves on density perturbations in the vicinity of a VLF antenna, Plasma Phys. Controlled Fusion, 29, 1511, 1987.

Groves, K. M., M. C. Lee, and S. P. Kuo, Spectral broadening of VLF radio signals traversing the ionosphere, J. Geophys. Res., 93, 14, 683, 1988.

Gurnett, D. A., and T. B. Burns, The low frequency of ELF emissions, J. Geophys. Res., 73, 7437, 1968.

Hayakawa, M., F. Lefeuvre, and J. L. Rauch, On the system of Aureol 3 satellite direction finding for ionospheric and magnetospheric ELF waves, Trans. Indt. Electron. Commun. Eng. Jpn., Sect. E, 73, 942, 1990.

Helliwell, R. A., Whistler and Related Jonospheric Phenomena, Stanford University Press, Stanford, Calif., 1965.

Inan, U. S., and T. F. Bell, Spectral broadening of VLF transmitter signals observed on DE 1; A quasi-electrostatic phenomenon? J. Geophys. Res., 90, 1771, 1985.

Lagoutte, D., F. Lefeuvre, and J. Hanasz, Application of bicoherence analysis in study of wave interactions in space plasma, J. Geophys. Res., 94, 435, 1989.

Lee, M. C., and S. P. Kuo, Production of lower hybrid waves and field-aligned density striations by whistlers, J. Geophys. Res., 89, 10,873, 1984.
Means, J. D., The use of the three-dimensional covariance matrix in analyzing the properties of plane-waves, J.Geophys. Res., 77, 5551, 1972.

Mosier, S. R., Poynting flux studies of hiss with the INJUN 5 satellite, J. Geophys. Res., 76, 1713, 1971.

Muzzio, J.L.R., and J.J. Angerami, OGO 4 observations of extremely low-frequency hiss, J. Geophys. Res., 77, 1157, 1972.

Parrot, M., World map of ELF/VLF emissions observed by a low-orbiting satellite, Ann. Geophys., 8, 135, 1990.

Rauch, J.L., J.C. Cerisier, J.J. Berthelier, F. Lefeuvre, N. Boud'ko, G. Michailova, and $O$. Kapustina, Attenuation bands and cut-off frequencies for ELF electromagnetic waves, Results of the ARCAD 3 Project and the Recent Programmes in the Magnetospheric and Ionospheric Physics, p. 445, CEPADUES Ed., Toulouse, 1985, France.

Riggin, D., and M. C. Kelley, The possible production of lower hybrid parametric instabilities by VLF ground transmitters and by natural emissions, J. Geophys. Res., 87, 2545, 1982.

Tanaka, Y., D. Lagoutte, M. Hayakawa, F. Lefeuvre, and S. Tajima, Spectral broadening of VLF transmitter signal and sideband structure observed on AUREOL 3 satellite at middle latitudes, J. Geophys. Res., 92, 7551, 1987.

Titova, E. E., V. I. Di, V. E. Yurov, O. M. Raspopov, V. Y. Trakhtengertz, F. Jiricek, and P. Triska, Interaction between VLF waves and the turbulent ionosphere, Geophys. Res. Lett., 11, 323, 1984.

V. Fiala, D. Lagoutte, F. Lefeuvre, and V. I. Sotnikov, CNRS/LPCE, 3A Avenue de la Recherche Sci., 45071 Orleans Cedex 2, France.

M. Mogilevsky, Space Research Institute (IKI) 117810 Moscow, USSR.

(Received April 28, 1990 ; revised January 29, 1991 ; accepted March 4, 1991.) 